Service social

\title{
Le phénomène de l'hébergement privé pour personnes âgées : peut-on éviter ou devrait-on l’aménager?
}

\section{Lorraine Brissette}

Volume 41, numéro 1, 1992

L'avenir des services ou services d'avenir

URI : https://id.erudit.org/iderudit/706558ar

DOI : https://doi.org/10.7202/706558ar

Aller au sommaire du numéro

\section{Éditeur(s)}

École de service social de l'Université Laval

ISSN

1708-1734 (numérique)

Découvrir la revue

Citer cet article

Brissette, L. (1992). Le phénomène de l'hébergement privé pour personnes âgées : peut-on éviter ou devrait-on l'aménager? Service social, 41(1), 67-83. https://doi.org/10.7202/706558ar
Résumé de l'article

Cet article aborde la problématique du développement anarchique et accéléré des ressources d'hébergement pour personnes âgées au cours des dix dernières années. Il décrit tout d'abord les caractéristiques de ces ressources pour ensuite analyser les causes de leur développement du point de vue des usagers et des intervenants. Il démontre la conjugaison quasi historique de deux facteurs principaux, soit l'augmentation démographique du poid des personnes du troisième mais surtout du quatrième âge et les changements dans la structure des revenus au Québec. Le déplacement progressif de la richesse vers les couches plus âgées de la population va entraîner un clivage dans cette population, clivage qui affectera le développement des ressources. L'article conclut sur le fait que la croissance du secteur privé dans le contexte social et économique actuel est inévitable, mais qu'elle soulève de nombreuses questions, dont celle de la responsabilité de l'État à l'égard du contrôle de la qualité des services qui sont donnés dans ce secteur. 
Lorraine Brissette, détentrice d'une maîtrise et étudiante au doctorat en service social (Laval). Elle s'intéresse particulièrement au domaine de la psychogériatrie.

\section{Le phénomène de l'hébergement privé pour personnes âgées : peut-on l'éviter ou devrait-on l'aménager?}

Lorraine Brissette

\section{Introduction}

Nous assistons depuis une dizaine d'années à la prolifération de résidences d'hébergement privées pour personnes âgées un peu partout au Québec. Ces résidences, non agréées pour plusieurs par le ministère de la Santé et des Services sociaux (MSSS), sont désignées sous des vocables variés qui représentent bien les diverses perceptions que nous avons d'elles : " foyers sans permis", "résidences pour retraités ou pour personnes autonomes ", "foyers clandestins ", " chambres et pension ", " résidences autofinancées avec services ", etc. Ces ressources privées à but lucratif ont pignon sur rue, font leur publicité dans les médias et vont jusqu'à se faire, dans certaines régions, une concurrence acharnée et même une guerre des prix.

L'État ne se désengage pas du secteur de l'hébergement des personnes âgées en perte d'autonomie en ce sens qu'il poursuit les engagements déjà pris, mais il ralentit de toute évidence le développement de nouvelles ressources d'hébergement et oriente ses efforts vers le maintien à domicile. En fait, il se désengage du secteur de l'hébergement des personnes âgées en perte d'autonomie moyenne et légère. Dans la réforme du ministre Côté, l'appellation centre d'accueil et d'hébergement est remplacée par celle de centre d'hébergement et de soins de longue durée. Ce changement peut nous indiquer que le MSSS entend orienter davantage 
son intervention vers le secteur de la santé, la vocation hébergement glissant lentement vers le secteur privé.

Comme l'indique très bien Vaillancourt, "La privatisation, pour nous, renvoie d'abord à un processus concret et non pas à un discours " (Vaillancourt, $1987:$ 9). Le développement du secteur de I'hébergement privé a fait l'objet de peu de recherches, le discours gouvernemental est très pauvre à ce sujet et les écrits des principaux groupes intéressés sont aussi peu abondants. Tout se passe comme si un "silence permissif » entourait le développement des ressources privées autofinancées pour les personnes âgées. Quelques organismes ont bien évidemment sonné l'alarme, telle I'Association des centres de services sociaux du Québec (ACSSQ) dans un document de réflexion intitulé « Les résidences d'hébergement privées non agréées, de la tolérance à l'intervention ". Mais de tels appels ne semblent pas avoir reçu d'échos. Devant ces silences, devant si peu d'actions, peut-on croire que le développement du réseau privé répond à certains besoins chez les usagers et les décideurs, et si oui, lesquels et pourquoi ? C'est ce que nous tenterons de clarifier dans cet article.

Nous mettrons en lumière l'ampleur du phénomène, les causes probables du développement du secteur privé du point de vue de l'usager et du point de vue démographique et économique. Nous conclurons par un questionnement sur les valeurs sociales mises en cause par le développement du secteur privé d'hébergement à but lucratif. Nous exclurons de notre exposé les ressources privées de type communautaire.

\section{Un phénomène d'importance}

Tout d'abord, essayons de cómprendre l'ampleur du phénomène et le contenu qu'il recouvre. Il faut souligner qu'aucun rapport de recherche ne fait état du nombre précis de résidences privées autofinancées. Selon une enquête menée par l'ACSSQ dans la région de l'Estrie et selon les extrapolations faites pour le Québec, il y aurait en 1989 environ 12000 places de résidences sans permis, soit approximativement autant que dans les ressources privées autofinancées avec permis, conventionnées et pavillonnées (Vaillancourt et Bourque, $1989: 60$ ). Les intervenants sociaux dans l'ensemble du Québec font état d'une croissance accélérée des ressources privées sans permis. Le rapport de l'ACSSQ est formel à cet effet : "Un réseau d'hébergement privé se développe présentement au Québec d'une façon accélérée et tout à fait anarchique » (ACSSQ, 1989 : 17). Selon une recherche effectuée par le Centre des services sociaux (CSS) Richelieu en 1984, sur 34 centres 
d'accueil privés dont 22 sans permis, il est ressorti que $71 \%$ de la clientèle hébergée fut évaluée comme étant non autonome. Une seconde constatation du rapport indique qu'il y a à toutes fins utiles peu de différence pour la qualité de vie des bénéficiaires entre des résidences avec ou sans permis (Vaillancourt, 1987 : 185).

Pourtant, les textes de loi ne peuvent être plus clairs. La Loi sur les services de santé et les services sociaux (LSSS) stipule que « nul ne peut exploiter un établissement s'il ne détient un permis permanent ou un permis temporaire délivré à cette fin par le ministre »(MSSS, 1971 : C48-94). La loi définit aussi que les centres d'accueil et d'hébergement (CAH) s'adressent en priorité aux personnes non autonomes (MSSS, 1979, art. 1K). Et la loi 120 du ministre Côté vient réaffirmer et même renforcer la mission des centres d'hébergement et de soins de longue durée à l'égard des personnes en perte d'autonomie importante (Gouvernement du Québec, loi 120 , chap. 1 , art. 83 ).

\section{Un développement permissif}

Au départ, les résidences privées autofinancées et sans permis ne sont pas illicites, puisqu'elles visent une clientèle autonome à laquelle elles offrent des services d'hôtellerie. Ces résidences deviennent progressivement illicites à mesure que la clientèle s'alourdit. On peut même affirmer que devant la concurrence du marché, de plus en plus de résidences accueillent, dès l'admission, des personnes en perte d'autonomie.

Ces résidences privées autofinancées sans permis du MSSS ont en général besoin d'un permis municipal d'exploitation commerciale. Ce permis régit ce que l'on qualifie de " permis de chambre et pension ". Ces résidences ne sont soumises à aucune forme de contrôle de la qualité, sauf en cas de plainte formelle si le MSSS exige une enquête précise. L'article 142 de la LSSS prévoit une procédure d'inspection précise. D'autres articles de la même loi prévoient également la possibilité pour le MSSS de faire évacuer ou de déplacer les personnes qui sont hébergées dans des résidences sans permis; les modalités y sont prévues et même les pénalités. Une amende de $2000 \$$ à 5000 \$ est prévue pour les individus et de $5000 \$$ à $10000 \$$ pour les corporations (MSSS, C48-137). Cependant, nous savons tous que ces recours légaux sont très rarement utilisés, uniquement pour les cas extrêmes où il y a plainte.

En fait, la législation existe, mais le MSSS n'y a pas consacré les ressources pour les mettre en application. II n'y a pas de contrôle systématique, rigoureux et régulier des ressources sans permis. L'opération pavillonnement réalisée en 1976 a ponctuellement 
effectué un ménage dans ce réseau parallèle, mais ce fut un " coup d'épée dans l'eau ", puisque le réseau s'est rapidement reconstitué. Le MSSS, implicitement, a choisi de ne pas mettre en application sa propre législation!

\section{Une extrême variabilité dans la qualité des ressources}

Les résidences privées sans permis sont extrêmement disparates; certaines $n^{\prime}$ accueillent que quelques bénficiaires, tandis que d'autres constituent des structures pouvant accueillir deux cents ou trois cents personnes.

Si nous prenons comme comparaison les services d'hébergement (gîte et couvert) offerts par les centres d'accueil et d'hébergement $(\mathrm{CAH})$ du secteur public, nous pouvons constater qu'il y a deux types de ressources privées. Certaines se situent très en dessous de ce "barème " du public en matière de qualité de services. D'autres, par contre, se situent très au-dessus sur le plan de la qualité des espaces, de l'alimentation, du site, de la qualité de la vie, etc. Certaines de ces résidences privées offrent des services de luxe à des personnes qui peuvent se les payer. L'éventail des services de base varie donc d'un extrême à l'autre en fonction des capacités financières des personnes.

Le coût de l'hébergement dans ces résidences varie considérablement. Cependant, il est important de souligner que la qualité de vie dans ces ressources n'est pas toujours dans un rapport proportionnel avec le prix payé. Certaines résidences de luxe font miroiter quantité de services à l'admission et ne les donnent qu'au compte-gouttes dans les faits, tandis que certaines ressources peu coûteuses peuvent offrir une bonne qualité d'encadrement. Dans certains milieux, le client paie surtout pour le décor, et « tout ce qui brille n'est pas or ! » Les services de soins et d'assistance quant à eux sont offerts parfois par la résidence; dans certains cas, ce sont les services du réseau public qui sont utilisés, et finalement ils peuvent être achetés dans une agence privée.

Il est nécessaire de signaler que la loi de l'offre et de la demande vient ici jouer en faveur du client. Selon notre expérience, dans les régions où les ressources d'hébergement sont abondantes, elles doivent offrir davantage de qualité pour se maintenir, sinon elles disparaissent à plus ou moins longue échéance. Notons aussi qu'en milieu rural et semi-urbain, la connaissance du milieu et la " réputation » de la qualité des ressources font en sorte d'éliminer plus facilement les mauvaises ressources.

Nous pouvons constater qu'il n'est pas possible de considérer l'ensemble des ressources autofinancées sans permis d'un même 
œil. Certaines répondent bien aux besoins des bénéficiaires et sont situées à proximité de leur milieu naturel. Il pourrait être important de conserver de telles ressources, elles pourraient être incorporées au système privé avec permis. Un minimum de contrôle des standards de qualité pourrait ainsi être assuré par l'État. Mais cette solution, à première vue réaliste, renvoie à un ensemble de problèmes de planification des ressources qui ne seront pas abordés dans cet article.

\section{Les causes du développement de l'hébergement privé selon le point de vue des intervenants et des usagers}

Le document de l'ACSSQ indique un certain nombre de causes à partir de l'expérience de très nombreux intervenants sociaux. L'une des premières causes mentionnées serait l'attente dans le réseau public d'hébergement. L'étude de d'Amours en 1986 pour I'Association des centres d'accueil du Québec (ACAQ) faisait état d'environ 7000 personnes en attente d'hébergement pour le réseau public (ACSSQ, 1989 : 11). " En contrepartie, l'accès et l'admission en résidence privée sont beaucoup plus faciles et ne sont pas soumis au contrôle qui prévaut dans le secteur public quant aux preuves de besoin d'hébergement "(ACSSQ, $1989: 11$ ).

Ce dernier point est essentiel et appelle des précisions. L'expérience de nombreux intervenants en CLSC démontre qu'une portion des personnes en perte d'autonomie moyenne et légère qui ont formulé une demande d'hébergement sont déjà placées dans le réseau privé lorsque la réponse à leur demande est acheminée. D'une part, la décision de recourir à l'hébergement a souvent été longue et laborieuse à prendre et lorsque le bénéficiaire est prêt, c'est souvent tout de suite qu'il veut une ressource. D'autre part, certaines familles et certaines personnes âgées réagissent mal à la longue et minutieuse évaluation exigée par le secteur public (CTMSP). Ils ne voient pas la nécessité de ces longues et indiscrètes enquêtes quand $c^{\prime}$ est si facile d'entrer chez madame $X$ ou madame $Y$ sans justifications.

Un troisième point, moins souvent discuté, mérite d'être mis en lumière. II s'agit de la preuve de besoin. La preuve faite par le système public est censée être très objective. Mais le ressenti de besoin du bénéficiaire est, lui, très subjectif. Si son besoin n'est pas jugé suffisamment urgent par le système (qui juge aussi en fonction de la disponibilité des services), il peut être vu comme très important par le bénéficiaire. À ce moment précis, un certain nombre de facteurs entrent en ligne de compte : le désir ou non de la per- 
sonne de demeurer chez elle, la présence ou non d'un réseau naturel, la solitude et l'insécurité, l'autonomie psychologique, les ressources de maintien à domicile offertes et le type d'intervention des praticiens sociaux.

Le praticien social est-il un évaluateur de demandes de placement ou un promoteur zélé de maintien à domicile? Si les ressources offertes ne répondent pas aux attentes, si l'intervenant n'a pas réussi dans sa "mission de promotion " du maintien à domicile, le bénéficiaire aura recours à ses propres solutions, soit d'aller chercher lui-même une ressource d'hébergement proche et disponible. Ne serait-ce pas une vue de l'esprit qui nous arrange bien de croire que " toutes les personnes âgées veulent demeurer à domicile "? Quant aux personnes âgées qui ne désirent pas demeurer à domicile même avec des services, leur faudra-t-il jouer le "jeu de la perte d'autonomie " pour obtenir ce qu'elles désirent?

Officiellement, la personne âgée et sa famille ne sont pas conseillées ou accompagnées dans cette démarche de recherche de ressources, ce qui serait sûrement moins dommageable que la situation actuelle. La position dans les faits est que si quelqu'un n'accepte pas les ressources proposées par le public, il n'a pas droit aux services sociaux de ce secteur. La position du sous-ministre Réjean Cantin en 1987 a été à cet effet très claire :

II nous semble donc important de demander à chacun d'entre vous d'aviser les professionnels(les) concernés(es) de vos établissements respectifs de s'abstenir de diriger, orienter, ou encore, référer officiellement ou officieusement des personnes en perte d'autonomie aussi bien physique que psychique dont l'état est relativement détérioré ou diminué, vers des ressources opérant sans permis. (MSSS, 1987)

Ceci nous renvoie à une question qui sera discutée plus loin au sujet des valeurs de notre société. Quelle place accordons-nous au choix des personnes âgées et au désir d'hébergement indépendamment de la gravité du besoin ?

D'autres facteurs viennent influencer le choix des personnes âgées de s'orienter vers le secteur privé. L'ACSSQ mentionne le manque de places dans le réseau des familles d'accueil dans certaines régions et la défection de certaines familles d'accueil qui ont rejoint le secteur privé. Soulignons aussi l'usage plus ou moins officiel de ces ressources qui est fait par un bon nombre de centres hospitaliers soit pour une période de convalescence à la suite d'un court séjour ou pour désengorger les hôpitaux. Ces pratiques ne sont pas le seul fait des intervenants sociaux comme beaucoup veulent le faire croire. Selon le CSS Richelieu, 
Certains ambulanciers réfèrent à leurs propres foyers illicites des clients desservis lors d'un transport ambulancier. Des infirmiers, préposés ou médecins, dans le cadre de leur travail en $\mathrm{CH}$, réfèrent dans leurs propres foyers ou dans un foyer tenu par un associé ou un conjoint. (CSSR, 1984 : 440)

Selon l'expérience de nombreux intervenants, il n'est pas rare de voir certains médecins en cabinet privé conseiller telle ou telle ressource à leurs patients âgés.

Une autre cause importante du développement du secteur privé peut être le désir des personnes âgées de continuer à vivre dans leur environnement social immédiat, alors que les places offertes dans le réseau public les obligeraient parfois à s'éloigner. Soulignons, et ceci est très important, que le réseau public n'offre pas de choix. II faut accepter la place disponible là où elle est, même si cela ne nous convient pas ou ne convient pas à la famille.

Finalement, et cette cause n'est pas à négliger, certaines personnes font le choix du secteur privé pour des motifs de qualité de vie. Dans bien des régions, les centres d'accueil et d'hébergement de construction plus ancienne ne disposent que de chambres doubles. Certaines personnes ou familles refusent ce compromis qu'elles jugent invivable, et la présence de plus en plus nombreuse de personnes âgées atteintes de déficits cognitifs vient aggraver la situation pour certaines personnes lucides. Elles préfèrent supporter le coût d'une ressource privée pouvant respecter leur intimité et pouvant présenter une qualité de vie supérieure à celle offerte dans le secteur public.

\section{Un développement accéléré par l'augmentation démographique des personnes âgées et les habitudes de consommation de services}

Comme chacun le sait, les facteurs démographiques sont éloquents. Selon les statistiques du dernier recensement :

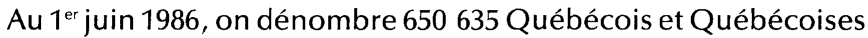
âgés de 65 ans et plus. Entre 1981 et 1986 leur nombre s'est accru de 81255 , soit de 2,7 \% par année en moyenne. [...] Le groupe des 65 ans et plus, qui représentait 4,8\% de la population en 1901 et $5,7 \%$ en 1951 en forme maintenant $10 \%$. Selon les projections de population, l'augmentation de la population âgée devrait se poursuivre de telle sorte qu'en 2006 le Québec compterait 13,7\% de personnes âgées... C'est le groupe le plus âgé ( 75 ans et plus) qui voit sa proportion s'accroître le plus fortement (de 3,7 \% de la population totale en 1986 à $6,3 \%$ en 2006). [...] C'est le groupe des 90 ans et plus qui augmente le plus entre 1981 et 2006 (34\%). (Gouvernement du Québec, 1989 : 397) 
Le Livre blanc du ministre Côté, intitulé " Une réforme axée sur le citoyen ", considère que l'on estime à environ $7 \%$ la proportion des personnes âgées qui vivent en milieu d'hébergement et de soins de longue durée. Ce taux place le Québec devant la France (5\%), la Grande-Bretagne (5\%); les États-Unis (5,3\%) et l'Australie $(5,9 \%)$.

Le gouvernement vise une diminution du taux d'institutionnalisation au Québec. Le coût de la santé chez les Québécois de 65 ans et plus est nettement supérieur à celui des citoyens américains et ontariens. Le Livre blanc attribue clairement ces résultats aux « caractéristiques du modèle d'intervention en vigueur et à la nature des services offerts. Ces éléments risquent même de peser plus lourd dans l'avenir sur le rythme de progression des dépenses que le vieillissement démographique » (Gouvernement du Québec, Livre blanc : 24).

En clair, les habitudes de consommation de services des personnes âgées, le style de pratique des intervenants, les investissements en ressources plus importants dans le secteur de l'hébergement que dans celui du maintien à domicile expliquent la situation.

Malgré ses efforts pour promouvoir le maintien à domicile, le MSSS constate que la diminution des taux d'hébergement est très lente. La norme actuelle du MSSS s'appuie sur des taux de 1,3\% pour les $65-74$ ans et de $10 \%$ pour les 75 ans et plus. Une application pure et simple de cette norme laisse présager un déficit approximatif de près de 9000 lits en l'an 2001 et de 27000 lits à peine dix ans plus tard.

Nous pouvons nous demander, si les places en hébergement public sont destinées en priorité aux personnes en perte d'autonomie sévère, où iront les personnes en perte d'autonomie moyenne ou légère. Nous pouvons faire l'hypothèse qu'elles iront en grand nombre vers le secteur privé. II ne faut pas oublier qu'au Québec, dans la culture des personnes âgées, et souvent de leur famille, le recours à l'hébergement en cas de perte d'autonomie est souvent considéré comme un droit social.

L'une des explications possibles de la croissance accélérée du secteur privé d'hébergement et de laisser-faire apparent de l'État pourrait-elle se situer dans la conjonction quasi historique de la croissance démographique du troisième et du quatrième âge et de l'émergence d'une classe moyenne retraitée?

Du point de vue des intervenants sociaux qui travaillent généralement avec des personnes en perte d'autonomie, le troisième âge est souvent associé à une image de pauvreté, de maladie, de fragilité, de vulnérabilité, de dépendance, etc. La liste des vocables 
qui caractérisent la clientèle du secteur public est très variée. Dans l'ensemble, elle définit une seule partie de la population âgée globale, mais elle définit surtout une cohorte d'âge. Les personnes âgées en besoin de services dans la décennie actuelle ne sont plus celles des années 70 .

\section{Une nouvelle réalité économique en émergence : l'enrichissement du troisième âge}

Si nous quittons le monde des services publics pour regarder ce qui se prépare dans le monde des affaires, nous aurons une idée beaucoup plus exacte de la réalité actuelle du troisième âge. La revue L'actualité dans sa section Commerce nous indiquait en 1989 que "les 50 ans et plus constituent depuis cette année le groupe de consommateurs numéro un dans le collimateur des publicitaires avec plus de la moitié des revenus disponibles." (L'actualité, 1989 : 138).

En fait, 1,5 million de Québécois contrôlent $55 \%$ du revenu personnel disponible. Selon Raymond Kerouack de la Banque Toronto Dominion : "Les 50 ans et plus représentent $25 \%$ de la population en 1986. Ce sera $34 \%$ en I'an 2000, soit dans moins de dix ans. Près de $75 \%$ de la valeur nette canadienne est déjà entre leurs mains. »(Gendron, 1990 : 65). Pour Jean Hurtubise, économiste consultant en habitation, " $c^{\prime}$ est la même chose en habitation. Les appartements luxueux apparus dans la dernière décennie ne s'achètent pas avec des revenus mais du capital. Les revenus sont entre les mains d'acheteurs plus âgés, qui ont des goûts, des besoins et les moyens de les satisfaire »(Gendron, $1990: 65)$. Pour Denis Chagnon d'Air Canada, " les voyageurs à cheveux gris ont de l'argent, du temps et beaucoup de flexibilité. Tous les transporteurs aériens leur portent une attention grandissante » (Gendron, 1990 : 66).

Finalement Jean Perrin, professeur de marketing à I'UQAM, résume la position de l'entreprise privée à l'égard du troisième âge : " C'est une clientèle fort mal connue. On voit encore les personnes âgées comme on voyait nos grands-parents. II faut oublier l'image du pauvre vieillard souffreteux et froussard, uniquement préoccupé de sa digestion et de sa pression artérielle. La réalité, c'est que $75 \%$ des retraités d'aujourd'hui sont en excellente santé. »(Gendron, 1990 : 23).

Il faut bien admettre que la maison payée, les enfants partis, l'accès à des tarifs réduits dans plusieurs services, la gratuité des médicaments chez les personnes de 65 ans et plus font que les préretraités et les retraités jouissent en grande partie d'un revenu 
plus élevé que par le passé. Selon les économistes, près de $40 \%$ de leurs dépenses vont à des produits de confort. II s'agit d'un phénomène lié au vieillissement de la classe moyenne qui a émergé après la Révolution tranquille. En réalité, il faut nuancer ces affirmations par le fait que " cette aisance nouvelle " se retrouve plutôt chez les jeunes retraités de 55 à 75 ans que chez les retraités du quatrième âge. De plus, nous devons considérer la question sous un autre angle. Nous savons qu la cohorte des 50 ans et plus s'enrichit proportionnellement davantage que celle des jeunes. Mais à l'intérieur de cette cohorte comment se répartit la richesse? Une chose est certaine, si l'on considère le profil de l'emploi depuis 30 ans, les hommes y sont nettement plus favorisés, ainsi que les couples. Les femmes seules représentent encore certainement le groupe pauvre de cette cohorte d'âge.

II semblerait, à l'appui de cette thèse de l'enrichissement du troisième âge, que ce groupe ait été moins atteint par la récession économique du début des années 80.

Le revenu moyen du ménage dont le soutien a 65 ans et plus n'a presque pas bougé entre 1980 et 1985 : de 21021 \$ en 1980, il est de $21035 \$$ en 1985. À cet égard, ces ménages sont favorisés puisque le revenu de ceux dont le soutien a moins de 65 ans a diminué au cours de la même période. Ce sont les ménages dont le soutien a moins de 25 ans qui subissent la réduction la plus sensible; leur revenu moyen est passé de 21056 à $17766 \$$, soit une baisse de $16 \%$. (Gouvernement du Québec, 1989 : 398)

La principale révélation de toutes les études menées par les firmes de marketing sur le marché des retraités, c'est qu'ils veulent des services, beaucoup de services, et adaptés à leurs besoins. Selon Jean Perrin :

Il y a une dimension macro-économique importante qu'on appréhende peu; nous nous dirigeons clairement vers une société de services. Or, les gens plus âgés sont des surconsommateurs de services. Ils ont déjà réalisé les investissements de base. Ils allouent une part de plus en plus importante de leurs revenus discrétionnaires au secteur des services au point où, en immobilier par exemple, l'aspect service devient aussi important que l'aspect immobilier pur. (Gendron, $1990: 67$ )

Nous pouvons faire l'hypothèse que l'émergence d'une nouvelle répartition sociale de la richesse en fonction de la classe sociale et de la strate d'âge a un rapport direct avec la croissance du secteur privé de l'hébergement. Plusieurs politiques sociales à l'égard du troisième âge ont un caractère $d^{\prime}$ 'universalité (pensions de vieillesse, assurance-hospitalisation, médicaments). Elles ont été mises en place à une époque où le troisième âge représentait à 
la fois un groupe peu nombreux et ayant sensiblement les mêmes caractéristiques : un faible niveau de scolarité et de revenu, de plus grandes attentes relativement au soutien de la famille et une préparation à la retraite très déficiente.

À partir des années 90, l'État s'attend à ce que le citoyen âgé paie davantage pour ses services, et ce, pour plusieurs raisons. Une bonne part des retraités plus aisés ne sont pas satisfaits par la qualité des services offerts par le secteur public et auraient la capacité de se payer des services plus luxueux. Par ailleurs, les groupes d'âge plus jeunes se sont appauvris et la classe moyenne dans son ensemble n'est pas prête à voir s'accroître le fardeau fiscal. Finalement, les rumeurs toujours persistantes sur le déficit éventuel des caisses de retraite et l'incapacité des générations futures à payer pour leurs aînés préparent un glissement vers une plus grande contribution des personnes âgées. Selon Jean Hurtubise, les consommateurs âgés ne veulent plus subir et prendre ce qu'on leur donne, ils veulent pouvoir choisir :

En habitation, on a commencé par construire des résidences adaptées et des manoirs pour aînés. Puis la dernière décennie a vu naître une série incroyable de formules de toutes sortes, du condo luxueux au quasi-hôpital en passant par les logements supervisés. Certains veulent la tranquillité, la nature, des services de santé, des installations sportives. D'autres veulent vivre dans l'agitation urbaine et s'installent au centre-ville, près de toutes les activités culturelles. (Gendron, $1990: 66$ )

Selon toutes ces statistiques fournies par les économistes, et selon le Livre blanc, $83 \%$ des personnes âgées à domicile seraient en relativement bonne santé, tandis qu'environ $7 \%$ sont totalement prises en charge par l'hébergement (CAH et CHSLD). Mais qu'en est-il des $10 \%$ qui restent ? Ces personnes sont théoriquement en perte d'autonomie à des degrés variables. Un certain nombre reçoit des services de maintien à domicile, en quantité souvent insuffisante. $\mathrm{D}^{\prime}$ autres se paient des services privés ou ont recours à l'hébergement privé avec ou sans accréditation et en conséquence avec ou sans contrôle de qualité. Et c'est là que le clivage des classes sociales apparaît le plus clairement.

\section{Le clivage économique de la population âgée}

Nous aimerions corriger une perception des faits apportés par Vaillancourt. Ce dernier affirme : "Nous pouvons nous demander si, à long terme, le développement des services d'hébergement à but lucratif n'est pas en train de mener à un clivage au sein de la 
population âgée non autonome ayant besoin d'hébergement... » (Vaillancourt, $1989: 64$ ).

En fait, le développement du secteur privé ne crée pas le clivage, celui-ci existe déjà. Ce développement vient tout simplement le mettre en lumière. II faudrait cesser de voir les personnes âgées comme un bloc monolithique où les classes sociales ne sont pas agissantes. S'il y a dualisation dans la société en fonction du revenu et du groupe d'âge, il y a aussi dualisation dans le groupe des citoyens plus âgés. D'un côté, nous trouvons les travailleurs retraités de classe moyenne ou de classe ouvrière ayant disposé de bonnes conditions de travail et de retraite. De l'autre côté, nous trouvons surtout des personnes qui n'ont pas travaillé, qui ont travaillé dans des emplois précaires et mal payés, qui n'ont pas de conjoint pour partager les dépenses de base. Ce sont en majorité des femmes.

Nous croyons qu'il faudrait remettre en question l'équation vieillesse $=$ pauvreté. Cette équation de moins en moins réelle dans les chiffres ne l'est plus depuis longtemps dans la perception que les personnes âgées ont d'elles-mêmes.

Les observations d'une recherche réalisée par l'auteur veulent que les personnes âgées, même à faibles revenus, ne se sentent pas nécessairement pauvres. En fait, le degré de satisfaction s'expliquerait davantage par l'écart entre leur vie active et leur retraite que par leur revenu selon des barèmes socialement admis. Pour beaucoup de femmes âgées la retraite est la première occasion où elles ont de l'argent personnel. Les revenus de la personne assistée socialement augmentent en fait avec le passage au troisième âge. E. Skutesky-Odier, cité dans cette recherche, explique fort bien ce phénomène (Brissette, 1983 : 38) :

Étant donné la vie difficile que la plupart des personnes de 65 ans et plus ont vécue en milieu rural, les structures de support de leur vieillesse leur conviennent. Elles leur permettent de vivre maintenant une vie satisfaisante pour la majeure partie d'entre elles... La différence entre ce que ces personnes âgées ont manqué autrefois et ce qu'elles reçoivent maintenant représente pour elles un changement fondamental et positif.

Les mêmes observations ont été faites par l'auteur en milieu urbain au début des années 80 . Ce n'est pas parce que les personnes âgées sont souvent économes et répugnent à la dépense qu'elles se voient pauvres et que nous pouvons les considérer comme telles. Il s'agit évidemment d'études menées sur la cohorte des personnes âgées des années 70. Les différences dans les conditions des revenus et de mise à la retraite de la cohorte des années 90 risquent de montrer là aussi une dualisation. 
Vaillancourt poursuit sur ce thème du clivage en introduisant le risque de marginalisation et de stigmatisation :

Cela ne risque-t-il pas de conduire à une situation où seules les personnes très défavorisées économiquement auraient accès aux services publics faute de moyens pour recourir au privé ? Si tel devenait le cas, qu'adviendrait-il de l'universalité des services? N'assisterions-nous pas à la constitution d'une forme de ghetto des personnes âgées " pauvres, nécessiteuses et indigentes » dans le réseau public ? Une telle marginalisation ne comporterait-elle pas son lot de stigmatisation et de remise en question des services publics en quantité et en qualité, par les non-bénéficiaires (Bell Lowther, 1988). (Vaillancourt, 1989 : 64)

Effectivement, il est probable que la proportion des personnes à faibles revenus ira en s'accroissant dans le secteur de l'hébergement public. Mais le principe d'universalité $n^{\prime}$ est pas touché. Tous ont un droit d'accès aux services publics, mais l'accessibilité est réservée aux personnes en forte perte d'autonomie et la durée d'attente peut être longue. En pratique, les citoyens qui choisissent le recours au privé (ils ne sont pas tous riches) ne reçoivent pas gratuitement certains services auxquels ils ont droit. Les citoyens qui ont recours au privé peuvent-ils à la fois voir respecter leur droit de choisir leur milieu de vie et leur droit à la gratuité des soins ? En ce sens, les personnes âgées pauvres ne sont pas touchées défavorablement par le développement du secteur privé. Le recours au privé laisse davantage de places libres dans le secteur public et accélère de ce fait l'accès au secteur public.

Quant à la stigmatisation, nous ne croyons pas qu'elle représente un risque majeur si nous tenons compte des observations faites précédemment sur la perception qu'ont les personnes âgées par rapport à leur pauvreté et si cette tendance se maintient. Considérons le risque de remise en question des services publics. Nous croyons que le rôle primordial de l'État serait de développer dans ses propres services des standards de qualité forçant le secteur privé à offrir des services de qualité semblable ou supérieure. Nous croyons que si la qualité de services dans le secteur public est bonne, les personnes âgées seront plus enclines à s'y orienter. Finalement, nous croyons que les personnes présentant une grande perte d'autonomie physique et particulièrement psychique auront tout intérêt à s'orienter vers le secteur public, quels que soient leurs revenus.

\section{La privatisation et la kyrielle de questions qu'elle soulève}

Nous pouvons nous interroger sur la nature des inquiétudes de Vaillancourt. Notre objectif de société est-il que les person- 
nes âgées hébergées dans le secteur public soient de provenance socio-économique variée ? L'objectif social à promouvoir est-il l'égalité ou la meilleure qualité de services possible dans le secteur public et par effet d'émulation dans le secteur privé ?

La question qui devrait davantage nous inquiéter est que les personnes défavorisées n'ayant pas accès à l'hébergement public faute de place ou parce qu'elles ne répondent pas aux critères se dirigent vers un hébergement privé de qualité douteuse et non contrôlée. Invoquer la marginalisation ou la stigmatisation des personnes qui " $n^{\prime}$ ont pas les moyens pour recourir au privé ", n'est-ce pas un faux problème ? Le vrai problème est ailleurs : il vient du non-contrôle de la qualité des services privés et, dans certains cas, de la faible qualité des ressources publiques. L'hébergement en chambre double en 1992, est-ce encore réaliste ?

Si l'État a la responsabilité que des services de qualité soient fournis aux personnes âgées en perte d'autonomie, est-ce à dire qu'il doit assumer lui-même tous ces services ? La responsabilité de l'État dans le contexte économique, démographique et politique actuel pourrait-elle être de faire en sorte que des services soient quantitativement et qualitativement assurés à tous ceux qui en ont besoin, tout en laissant une marge de choix aux citoyens par rapport à leur style de vie ? Cette position pourrait-elle résoudre en partie le questionnement sur les valeurs dans lequel comme société nous sommes placés?

Tout le débat ou l'absence de débat social sur la question de la privatisation est teinté de valeurs. Et pourtant, bien peu de personnes semblent soucieuses d'ouvrir ces questions sur la place publique. Elles pourraient s'énoncer ainsi : dans quelle mesure les réticences de l'État et des intervenants à l'égard de la collaboration avec le secteur privé n'est-elle pas empreinte de principes judéochrétiens du type : "L'argent corrompt ", "On doit servir les autres d'abord par altruisme et non par intérêt »? Mais est-ce la motivation de l'action qui compte ou la qualité et la pertinence de cette action ? Il est clair qu'une motivation financière sans contrôle extérieur peut engendrer la médiocrité. Sommes-nous prêts à faire le pari du contrôle ou continuerons-nous à nier la réalité ?

Jusqu'où notre société doit-elle promouvoir ou peut-être " imposer " le maintien à domicile, puisque nous avons décidé socialement que c'était la meilleure solution ? Comment considérons-nous les choix individuels de vie des personnes du troisième âge ? Doit-on pénaliser ceux qui n'ont pas choisi la voie officielle (maintien à domicile et hébergement public) en ne leur donnant pas accès aux services du réseau public et en ne contrôlant pas la qualité des ressources privées ? Le principe de liberté indi- 
viduelle est-il compatible avec les principes d'universalité, d'accessibilité et de continuité ?

En bref, sommes-nous prêts à accepter cette nouvelle réalité qui émerge, celle d'une génération de personnes âgées mieux informées, plus exigeantes, qui veut pouvoir choisir et peut-être nous contester? Nous devons pouvoir envisager qu'un net clivage existera dans le groupe des personnes âgées entre les classes sociales. Nous ne pourrons plus envisager bien confortablement des services uniformes, car la réalité de cette clientèle sera et est déjà multiforme.

La multiplicité des valeurs dans notre société doit nous amener à trouver des formules variées et souples pour fournir des services en complémentarité entre le public et le privé. Comment sauronsnous respecter et même encourager la volonté de faire des choix chez la personne âgée, et ce, jusqu'au bout ? Le recours au secteur privé est souvent le résultat d'un choix et toute la vie à l'intérieur de ces ressources porte le sceau de la négociation. Dans le secteur de I'hébergement privé, la personne âgée négocie ses services et le maintien de sa présence (pour son avantage ou son désavantage). Par notre expérience, nous avons pu constater que la personne qui a encore une bonne autonomie psychique ou qui jouit de la présence d'un réseau naturel vigilant a un pouvoir de négociation, là où les ressources privées sont abondantes.

Cela nous amène évidemment à nous interroger sur la place et le pouvoir réel de la personne âgée prise en charge par les services publics. Le secteur public, jusqu'au milieu des années 80 largement majoritaire dans I'hébergement au Québec, devra se requestionner devant l'émergence du secteur privé. L'émulation devra exercer des pressions sur les deux secteurs. La principale critique à adresser à l'État n'est pas d'avoir développé le secteur privé, puisque les conditions socio-économiques nous y amèneraient tôt ou tard. C'est plutôt l'absence de responsabilisation de l'État par rapport au développement anarchique et sans contrôle de qualité des ressources privées. Il s'agit à notre avis d'un grave péché d'omission.

\section{Conclusion}

En guise de conclusion, nous pouvons déjà discerner les pistes qu'entend suivre l'État en rapport avec le développement du secteur privé. Le Livre blanc du ministre Côté a présenté de nombreuses propositions. Mais nous savons bien qu'une partie seulement de ces énoncés d'intention seront appliqués. Le Livre blanc 
propose entre autres de donner des mandats élargis aux CLSC pour fournir des services de maintien à domicile dans les résidences privées, pour recenser, visiter les personnes hébergées en " foyers clandestins " et évaluer leurs besoins. II propose aussi d'acheter des places ou des services dans les centres d'hébergement privés pour combler les 7000 places évaluées comme manquantes.

Dans les faits, le texte final de la loi stipule que la régie régionale peut attribuer à une résidence privée d'hébergement une allocation financière pouvant varier selon la matière des services offerts (Gouvernement du Québec, loi 120, chap. III, art. 454). Nous ouvrons peut-être ici la porte à " I'achat de places " proposé par le Livre blanc. Quant à la surveillance de la qualité des foyers sans permis, le texte de loi ne fait mention que des pouvoirs d'inspection du ministre présents dans les textes de la loi antérieure (loi 120, chap. VIII, art. 489). II ne fait nullement état d'un suivi et d'un contrôle devant être assurés par les CLSC. Seule la régie régionale y est mentionnée comme devant conseiller le ministre dans l'attribution, les modifications, les suspensions et révocations de permis.

La toute récente loi modifiant la Loi sur les services de santé et services sociaux ne vient nullement répondre à nos inquiétudes en matière de contrôle de qualité des services dans le secteur privé avec ou sans accréditation. En resterons-nous encore à un simple contrôle administratif avec les faiblesses que nous lui connaissons ? Quant aux droits, aux recours, aux plaintes, à l'accès au dossier dont il est largement fait mention dans la loi 120, comment l'État étendra-t-il sa réforme à tous les citoyens ? Nous sommes en droit de nous interroger.

\section{Références bibliographiques}

ACSSQ (1989). Les résidences d'hébergement privé non agréées, de la tolérance à l'intervention (décembre).

L'ACTUALITÉ (1989). "L'âge d'or et l'argent », avril.

BRISSETTE, Lorraine (1983). Influence de la fréquentation des centres de jour sur les réseaux de support naturel des personnes âgées et leurs stratégies de solution de problème, thèse de maîtrise, Université Laval.

CSSR (1984). Afin que demain. Longueuil.

GENDRON, Louise (1990). "Les vieux ne sont plus ce qu'ils étaient ", Revue commerciale (septembre).

Gouvernement du Québec (1971). Loi sur les services de santé et les services sociaux, chap. 48. 
Gouvernement du Québec (1991). Projet de loi 120, Loi sur les services de santé et les services sociaux et modifiant diverses dispositions législatives.

Gouvernement du Québec (1989). Le Québec statistique, 59e édition.

Gouvernement du Québec (1990). Une réforme axée sur le citoyen, MSSS.

PLAMONDON, Denis (1991). "Vers un modèle de services sociosanitaires alternatifs », revue Intervention (octobre), $\mathrm{n}^{\circ} 90$.

SKUTESKY-ODIER, E. "La personne âgée en milieu rural et son revenu ", Cahiers de l'ACFAS $n^{\text {os }}$ 13-2-12.

VAILLANCOURT, Yves (1987). La privatisation des services sociaux. Québec : Les Publications du Québec, annexe 37 de la Commission Rochon. 